\title{
LETTER
}

Lymphoma

\section{A proposal for a new staging system for extranodal natural killer T-cell lymphoma: a multicenter study from China and Asia Lymphoma Study Group}

\author{
Huangming Hong ${ }^{1,2} \cdot$ Yexiong $\mathrm{Li}^{3} \cdot$ Soon Thye $\mathrm{Lim}^{4} \cdot$ Chaoyong Liang ${ }^{5} \cdot \mathrm{He}$ Huang ${ }^{1} \cdot$ Pingyong $\mathrm{Yi}^{6} \cdot \mathrm{TaO} \mathrm{Wu}^{7}$. \\ Xin $\mathrm{Du}^{8} \cdot$ Mingzhi Zhang ${ }^{9}$. Jinghua Wang ${ }^{10}$. Jun Zhu ${ }^{11}$. Ting Liu ${ }^{12} \cdot$ Fanyi Meng ${ }^{13} \cdot$ Gang Wu $^{14} \cdot$ Ye Guo $^{15}$.

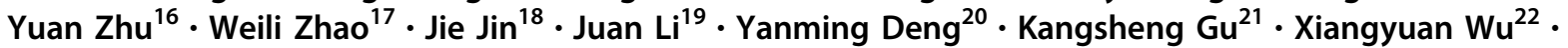 \\ Xiaoyan $\mathrm{Ke}^{23} \cdot$ Derong $\mathrm{Xie}^{2} \cdot$ Daren $\mathrm{Lin}^{24} \cdot$ Zhigang Peng ${ }^{25}$. Junxin $\mathrm{Wu}^{26} \cdot \mathrm{Qing} \mathrm{Liu}^{27} \cdot$ Won Seog Kim ${ }^{28} \cdot$ Tongyu Lin $^{1}$
}

Received: 19 August 2019 / Revised: 16 January 2020 / Accepted: 3 February 2020 / Published online: 17 February 2020

(c) The Author(s) 2020. This article is published with open access

\section{To the Editor:}

Extranodal natural killer T-cell lymphoma (ENKTL) is a distinct entity in the World Health Organization (WHO) classification. Patients suffering from this type of disease have poor survival outcomes [1-7]. However, using the Ann Arbor staging system (AASS), a routine lymphoma staging system, most ENKTL patients are categorized as early stage, which is inconsistent with their poor survival [1, 8-12]. Since the AASS has limited utility in the prognostication and treatment decision making for patients with ENKTL, this study aimed to develop a new staging system specific for ENKTL that can effectively identify patients with poor prognosis and provide information for personalized therapy.

There were three components of this study: a training cohort consisting of two stages and a validation cohort. In the training cohort, we first conducted a retrospective study of ENKTL patients treated with cyclophosphamide, doxorubicin, vincristine, and prednisolone (CHOP) or CHOP-like regimens or radiotherapy (RT) alone between Jan 1999 and Jun 2008 in 19 hospitals in China, with the aim to identify high-risk factors for proposing the new

These authors contributed equally: Huangming Hong, Yexiong Li, Soon Thye Lim, Chaoyong Liang, He Huang

Supplementary information The online version of this article (https:// doi.org/10.1038/s41375-020-0740-1) contains supplementary material, which is available to authorized users.

Tongyu Lin

tongyulin@hotmail.com

Extended author information available on the last page of the article staging system: the Chinese Southwest Oncology Group and Asia Lymphoma Study Group ENKTL (CA) system. Second, we conducted a prospective study of patients treated with asparaginase-based regimens or RT between Jul 2008 and Dec 2012 in the same 19 hospitals to determine whether the CA system was suitable in the era of asparaginase-based treatment. Based on the results of retrospective study and prospective study, the final form of the CA system was established. To validate the results from the training cohort, we performed an independent validation cohort study between Jan 2010 and Dec 2017 with the same inclusion and exclusion criteria using data obtained from Samsung Medical Center in South Korea, the National Cancer Center in Singapore and five hospitals in China, that were not included in the training cohort. The inclusion criteria were: (1) central pathologically confirmed diagnosis of ENKTL according to the 2008 WHO classification of lymphomas; (2) treatment with chemotherapy with or without radiotherapy or with RT alone with curative intent; and (3) availability of all clinical data required for staging and survival analyses. Local invasiveness was defined as invasion of the bone or perforation or invasion of the skin or paranasal extension as previously reported [9]. Regional lymph node involvement was defined as the invasion of lymph nodes corresponding to $\mathrm{N} 1, \mathrm{~N} 2$, or $\mathrm{N} 3$ of the primary lesion in accordance with 2002 TNM classification of the American Joint Committee on Cancer. The nasal and nonnasal types were defined based on the involvement of the nasal area, as reported previously [5]. The Institutional Review Board of Sun Yat-sen University Cancer Center (Guangzhou, China) reviewed and approved all aspects of this study. The statistical methods are summarized in Supplementary Appendix. 
The CONSORT flow of the study was shown in Supplementary Fig. 1. The training cohort included 1168 patients. Table 1 summarizes the characteristics of the patients. In the retrospective study, patients with lesions confined to the nasal cavity or nasopharynx without local tumor invasiveness showed a superior 5-year overall survival (OS) rate than patients with lesions complicated by local tumor invasiveness $(59.7 \%$ vs. $48.1 \%, P=0.01)$. Those with nonnasal-type disease without lymph node involvement had a lower 5-year OS rate than those with nasal-type disease without lymph node involvement (56.7\% vs. $40.1 \%, \quad P<0.01)$. Thus, patients with nonnasal-type disease or lesions confined to the nasal cavity or nasopharynx complicated by local tumor invasiveness were classified as stage II. Patients harboring lesions with regional lymph node involvement exhibited a lower 5-year OS rate than those without regional lymph node involvement $(34.7 \%$ vs. $52.3 \%, P<0.01)$. Thus, patients harboring lesions with regional lymph node involvement were categorized as stage III. Further, patients with non-regional lymph node involvement or lymph node involvement on both sides of the diaphragm or disseminated disease did not show 5-year OS rate difference (26.5\% vs. $27.1 \%$ vs. $25.7 \%, P=0.342$ ), these patients were classified as stage IV. Thus, we propose that the CA be stratified as follows: stage I, lesions confined to the nasal cavity or nasopharynx without local invasiveness and lymph node involvement; stage II, nonnasal-type disease or lesions confined to the nasal cavity or nasopharynx with local invasiveness without lymph node involvement; stage III, lesions with regional lymph node involvement; and stage IV, involvement of nonregional lymph node or lymph nodes on both sides of the diaphragm or disseminated disease. In the retrospective study involving patients who received CHOP-like treatment, the CA effectively discriminated survival; we then performed the prospective study and found that CA also effectively in discriminating the survival of patients who received asparaginase-based treatment.

According to the AASS, the patient distribution in the training cohort from stages I through IV was $61.8 \%, 20.4 \%$, $5.7 \%$, and $12.1 \%$, respectively. However, according to the CA system, the distribution was $27.4 \%, 35.2 \%, 18.7 \%$, and $18.7 \%$, respectively, from stages I through IV (Supplementary Table 1).

The 5-year OS rate for the training cohort was $52.4 \%$ (95\% confidence interval (CI) 48.9-55.9), and the 5-year progression-free survival (PFS) rate was $49.0 \%$ (95\% CI 45.5-52.5). In the training cohort, the CA system exhibited good patient stratification, with 5-year OS rates of $70.8 \%, 53.1 \%, 38.6 \%$, and $29.9 \%$ for stages I through IV $(P<0.001)$, respectively, and 5-year PFS rates of $67.5 \%$, $52.6 \%, 35.6 \%$, and $21.1 \%(P<0.001)$, respectively.
Alternatively, the 5-year OS rates were $60.7 \%, 42.9 \%$, $17.5 \%$, and $32.1 \%$ for AASS stages I through IV $(P<$ $0.001)$, respectively, and the 5-year PFS rates were $59.1 \%, 38.3 \%, 10.4 \%$, and $22.4 \%(P<0.001)$, respectively (Fig. 1a-d). For patients receiving CHOP-like treatment who were diagnosed with $\mathrm{CA}$ stages I through IV, the 5-year OS rates also showed reasonable declines; however, when diagnosed using the AASS, the survival of staging IV was better than that of staging III (Supplementary Fig. 2a, b). This result was similar for patients receiving asparaginase-based treatment (Supplementary Fig. 2c, d).

Patients from Singapore $(n=114)$, South Korea $(n=$ $102)$, and China ( $n=769)$ were included in the independent validation cohort, the 5-year OS rate was $50.4 \%$ (95\% CI 46.1-54.7), and the 5-year PFS rate was $46.0 \%$ (95\% CI 41.7-50.3) in this cohort. The CA system effectively stratified the OS and PFS for all 985 patients. (Fig. 1e-h).

In the receiver operating characteristic (ROC) analysis, the CA system better discriminated survival than the AASS in the training cohort (area under the curve (AUC), 0.68 vs. $0.60, P=0.013$ ) and the validation cohort (AUC, 0.70 vs. $0.61, P=0.032$ ). For all 842 patients who received asparaginase-based treatment in the training cohort and validation cohort, the prognostic index of natural killer lymphoma (PINK) [13] could stratify the survival according to different risk groups (Supplementary Fig. 3). However, the ROC analysis of CA system was superior than PINK (AUC, 0.71 vs. 0.64, $P=0.031$ ). For the 842 asparaginase-based treatment patients, for CA stage I, the 5-year OS rates for RT and chemotherapy combined with radiotherapy were similar (81.6\% vs. $85.8 \%, P=0.248$ ). For CA stage II, RT resulted in the lowest 5-year OS rate of $70.1 \%$, while concurrent chemoradiotherapy (CCRT), induction chemotherapy followed by radiotherapy, or concurrent chemotherapy $(\mathrm{CT}+\mathrm{CCRT} / \mathrm{RT})$ and CCRT followed by adjuvant chemotherapy (CCRT + CT) showed similar 5-year OS rates (75.2\%, 82.3\%, and 76.7\%, respectively, $P=0.754)$. Patients with CA stage III receiving $\mathrm{CT}+\mathrm{CCRT} / \mathrm{RT}$ exhibited the highest 5-year OS rate of $73.5 \%, \mathrm{CCRT}+$ CT and CCRT had moderate 5-year OS rates of $67.0 \%$ and $55.3 \%$, respectively, and those receiving $\mathrm{RT}$ had the lowest 5 -year OS of $32.3 \%(P<0.001)$. For CA stage IV, patients receiving autologous transplantation after chemotherapy did not show superior survival than those who did not $(57.1 \%$ vs. $23.5 \%, P=0.174)$ (Supplementary Fig. 4).

The AASS could not reasonably stratify the survival of ENKTL patients, since the survival of patients with stage IV was better than that of stage III. Less than $10 \%$ of patients were classified as stage III by the AASS, the 
Table 1 Comparison of characteristics between the validation and training cohorts.

\begin{tabular}{lll}
\hline Characteristic & $\begin{array}{l}\text { Training cohort } \\
n=1168,\end{array}$ & $\begin{array}{l}\text { Validation } \\
\text { cohort } n=985, \\
\text { no. }(\%)\end{array}$ \\
& no. $(\%)$ \\
\hline
\end{tabular}

Age, years

$\leq 60$

$>60$

Sex

Male

Female

ECOG PS

0-1

2-4

"B" symptoms

Absent

Present

Serum LDH level

Normal

Elevated

IPI

$0-1$

2-5

NK prognostic index

1-2

3-4

Local invasiveness

Absent

Present

Nonnasal type

Yes

No

Regional lymph-node involvement

Absent

Present

Bone marrow

involvement

Absent
Present

Ann Arbor staging system stage

$$
\text { I-II }
$$

III-IV

WBC count

$$
>4000 \text { per } \mathrm{mm}^{3}
$$$$
<4000 \text { per } \mathrm{mm}^{3}
$$

985 (84.3)

837 (85.0)

183 (15.7)

148 (15.0)

804 (68.8)

650 (66.0)

364 (31.2)

335 (34.0)

1061 (90.8)

896 (91.0)

87 (9.2)

89 (9.0)

570 (48.8)

455 (46.2)

598 (51.2)

$530(53.8)$

$822(70.5)$

680 (69.0)

346 (29.6)

305 (31.0)

869 (74.4)

716 (72.7)

299 (25.6)

269 (27.3)

690 (59.1)

554 (56.2)

478 (40.9)

431 (43.8)

688 (58.9)

$562(57.1)$

480 (41.1)

423(42.9)

162 (13.9)

147 (14.9)

1006 (86.1)

838 (85.1)

950 (81.3)

813 (82.5)

218 (18.7)

172 (17.5)

1093 (93.6)

913 (92.7)

75 (6.4)

72 (7.3)

961 (82.3)

822 (83.5)

207 (17.7)

163 (16.5)

954 (81.7)

824 (83.7)

$\mathrm{Hb}$ level

$$
\begin{aligned}
& >110 \mathrm{~g} / \mathrm{L} \\
& <110 \mathrm{~g} / \mathrm{L}
\end{aligned}
$$$$
214 \text { (18.3) }
$$

161 (16.3)

945 (80.9)

800 (81.2)

223 (19.1)

\begin{tabular}{|c|c|c|c|}
\hline Characteristic & $\begin{array}{l}\text { Training cohort } \\
n=1168 \\
\text { no. }(\%)\end{array}$ & $\begin{array}{l}\text { Validation } \\
\text { cohort } n=985, \\
\text { no. }(\%)\end{array}$ & $P$ \\
\hline Platelet count & & & 0.877 \\
\hline$>100,000$ per $\mathrm{mm}^{3}$ & 1070 (91.6) & $900(91.4)$ & \\
\hline$<100,000$ per $\mathrm{mm}^{3}$ & $98(8.4)$ & $85(8.6)$ & \\
\hline $\begin{array}{l}\text { Absolute } \\
\text { lymphocyte count }\end{array}$ & & & 0.165 \\
\hline$>1000$ per $\mathrm{mm}^{3}$ & $808(69.2)$ & $653(66.3)$ & \\
\hline$<1000$ per $\mathrm{mm}^{3}$ & $360(30.8)$ & $332(33.7)$ & \\
\hline Serum albumin level & & & 0.240 \\
\hline$>35 \mathrm{~g} / \mathrm{L}$ & $965(82.6)$ & $794(80.6)$ & \\
\hline$<35 \mathrm{~g} / \mathrm{L}$ & $203(17.4)$ & $191(19.4)$ & \\
\hline \multicolumn{4}{|l|}{ Treatment regimens } \\
\hline Anthracycline-based & & & $<0.001$ \\
\hline RT alone & $200(23.1)$ & $20(4.5)$ & \\
\hline CHOP & $502(58.1)$ & $318(71.1)$ & \\
\hline CHOP-like & $162(18.8)$ & 109 (24.4) & \\
\hline Asparaginase-based & & & 0.070 \\
\hline RT alone & $82(30.0)$ & $186(34.6)$ & \\
\hline SMILE-like & $49(16.1)$ & $73(13.6)$ & \\
\hline Platinum containing & $173(56.9)$ & $279(51.8)$ & \\
\hline
\end{tabular}

0.498

0.500
Table 1 (continued)

Local invasiveness was defined in the text.

ECOG PS eastern cooperative oncology group performance status, $L D H$ lactate dehydrogenase, IPI International Prognostic Index, NK natural killer, $W B C$ white blood cell, $H b$ hemoglobin, $R T$ radiotherapy, $C H O P$ cyclophosphamide, doxorubicin, vincristine, and prednisolone, SMILE dexamethasone, methotrexate, ifosfamide, L-asparaginase, and etoposide.

highly unbalanced distribution may produce unavoidable survival bias. Yan et al. [14] recently suggested a TNM staging system for ENKTL. However, that study was performed at a single-center, focused only on nasal patients, and the majority of patients enrolled received anthracycline chemotherapy, thus limiting the generalizability of that staging system. Currently, PINK is used to predict prognosis, but factors including the stage and lymph node involvement in this index are traditionally classified as part of the staging system. Thus, the application of PINK should depending on patient's general characteristics and staging factors. The CA system was established based on anatomic factors and can efficiently classify patients into different stages. The anatomic factors can be conveniently examined through imaging examinations. Moreover, the ROC analysis suggested that CA staging system is superior to AASS and PINK.

In terms of guiding contemporary asparaginase-based treatment, our study recommended RT for stage I; chemotherapy combined with radiotherapy for stage II; CT + CCRT/RT for stage III; and intensive chemotherapy for 
Fig. 1 The OS and PFS of the training cohort and the validation cohort. a OS staging using the AASS for the training cohort. b OS staging using the CA system for the training cohort. c PFS staging using the AASS for the training cohort. d PFS staging using the CA system for the training cohort. e OS staging using the AASS for the validation cohort. $f$ OS staging using the CA system for the validation cohort. $\mathrm{g}$ PFS staging using the AASS for the validation cohort. h PFS staging using the CA system for the validation cohort.
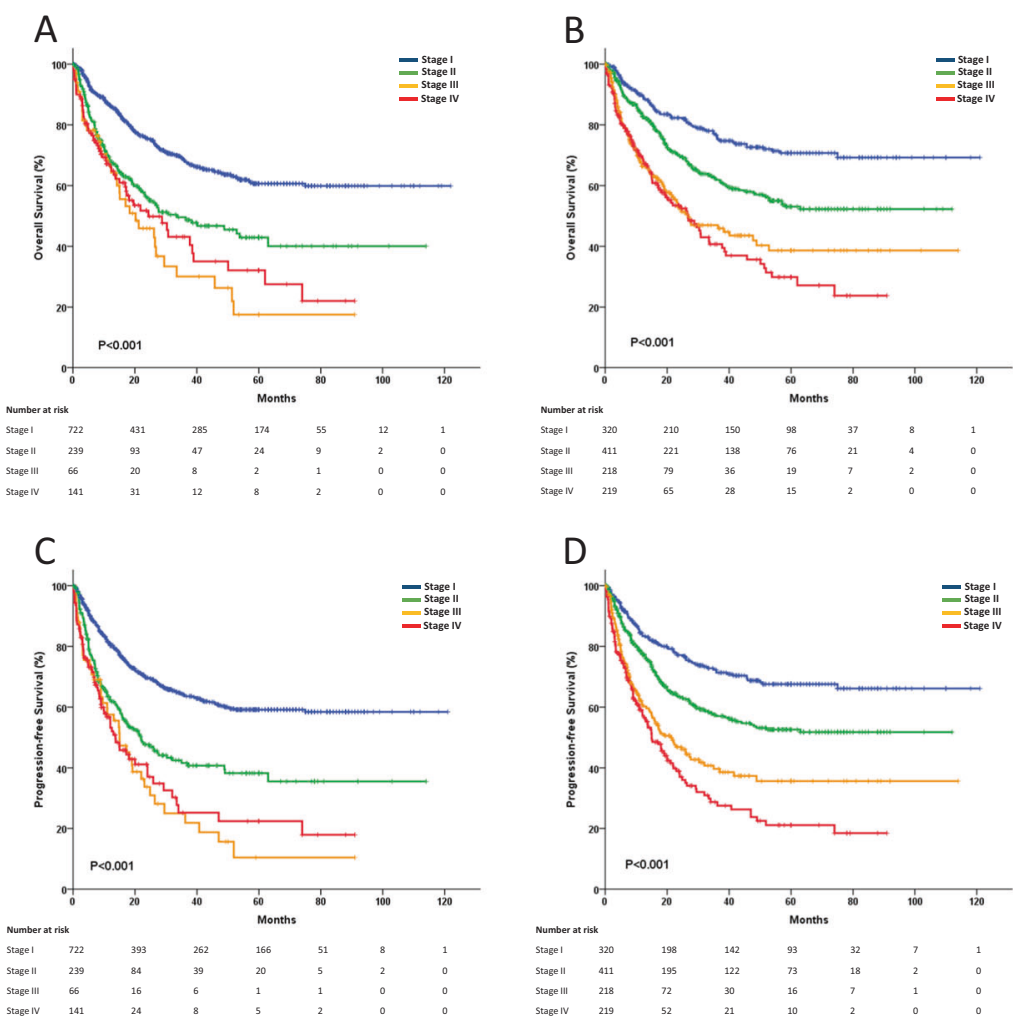

D
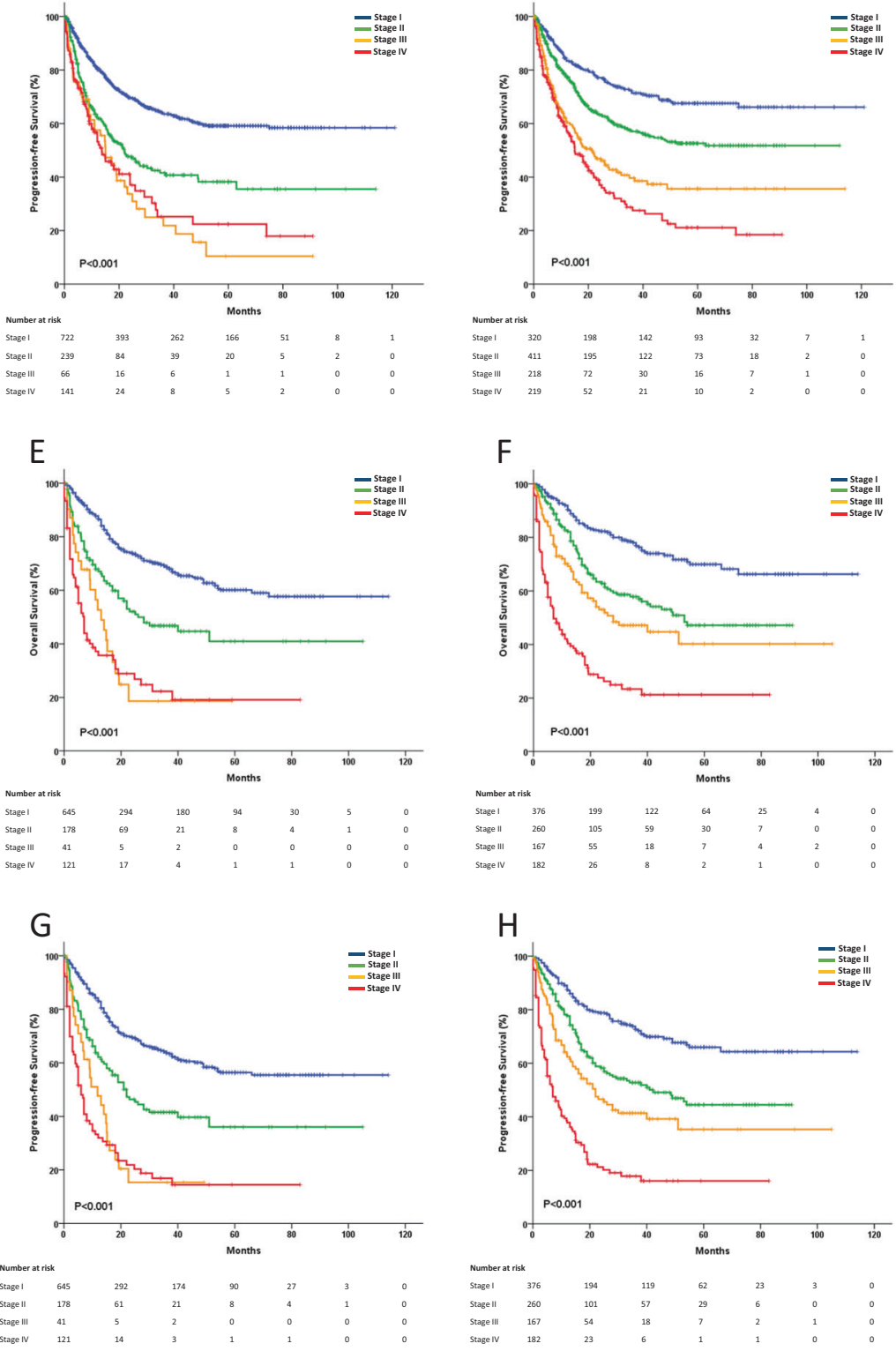

stage IV. However, the treatment regimens in our study were varied, validation in a second data set is needed, most preferable in a prospective manner.
The CA system demonstrated better survival discrimination than the AASS, and might add prognostic value and inform treatment decisions for ENKTL. It is crucial to 
accurately identify high-risk patients to improve outcomes in this subset of lymphoma.

Acknowledgements This work is supported by Guangdong Science and Technology Department (grant numbers 2017B020227002 and 2017A020215030). We thank all the patients, their families, and the institutions for supporting this study. We thank the committee on malignant lymphoma from the Chinese Southwest Oncology Group and the Asia Lymphoma Study Group.

Author contributions TYL designed the research. TYL and HMH collected and analyzed the data. HMH, CYL, and HH, wrote the article. All authors provided study materials or patients and approved the article.

\section{Compliance with ethical standards}

Conflict of interest The authors declare that they have no conflict of interest.

Publisher's note Springer Nature remains neutral with regard to jurisdictional claims in published maps and institutional affiliations.

Open Access This article is licensed under a Creative Commons Attribution 4.0 International License, which permits use, sharing, adaptation, distribution and reproduction in any medium or format, as long as you give appropriate credit to the original author(s) and the source, provide a link to the Creative Commons license, and indicate if changes were made. The images or other third party material in this article are included in the article's Creative Commons license, unless indicated otherwise in a credit line to the material. If material is not included in the article's Creative Commons license and your intended use is not permitted by statutory regulation or exceeds the permitted use, you will need to obtain permission directly from the copyright holder. To view a copy of this license, visit http://creativecommons. org/licenses/by/4.0/.

\section{References}

1. Kim TM, Lee SY, Jeon YK, Ryoo BY, Cho GJ, Hong YS, et al. Clinical heterogeneity of extranodal NK/T-cell lymphoma, nasal type: a national survey of the Korean Cancer Study Group. Ann Oncol. 2008;19:1477-84.

2. Lee J, Suh C, Park YH, Ko YH, Bang SM, Lee JH, et al. Extranodal natural killer T-cell lymphoma, nasal-type: a prognostic model from a retrospective multicenter study. J Clin Oncol. 2006;24:612-8
3. Bi XW, Li YX, Fang H, Jin J, Wang WH, Wang SL, et al. Highdose and extended-field intensity modulated radiation therapy for early-stage NK/T-cell lymphoma of Waldeyer's ring: dosimetric analysis and clinical outcome. Int J Radiat Oncol Biol Phys. 2013;87:1086-93.

4. Kim SJ, Park S, Kang ES, Choi JY, Lim DH, Ko YH, et al. Induction treatment with SMILE and consolidation with autologous stem cell transplantation for newly diagnosed stage IV extranodal natural killer/T-cell lymphoma patients. Ann Hematol. 2015;94:71-78.

5. Suzuki R, Suzumiya J, Yamaguchi M, Nakamura S, Kameoka J, Kojima $\mathrm{H}$, et al. Prognostic factors for mature natural killer (NK) cell neoplasms: aggressive NK cell leukemia and extranodal NK cell lymphoma, nasal type. Ann Oncol. 2010;21:1032-40.

6. Kwong YL, Kim WS, Lim ST, Kim SJ, Tang T, Tse E, et al. SMILE for natural killer/T-cell lymphoma: analysis of safety and efficacy from the Asia Lymphoma Study Group. Blood. 2012;120:2973-80.

7. Bi XW, Jiang WQ, Zhang WW, Huang JJ, Xia Y, Wang Y, et al. Treatment outcome of patients with advanced stage natural killer/ T-cell lymphoma: elucidating the effects of asparaginase and postchemotherapeutic radiotherapy. Ann Hematol. 2015;94:1175-84.

8. Cheung MM, Chan JK, Lau WH, Ngan RK, Foo WW. Early stage nasal NK/T-cell lymphoma: clinical outcome, prognostic factors, and the effect of treatment modality. Int J Radiat Oncol Biol Phys. 2002;54:182-90.

9. Kim TM, Park YH, Lee SY, Kim JH, Kim DW, Im SA, et al. Local tumor invasiveness is more predictive of survival than International Prognostic Index in stage $\mathrm{I}(\mathrm{E}) / \mathrm{II}(\mathrm{E})$ extranodal NK/ T-cell lymphoma, nasal type. Blood. 2005;106:3785-90.

10. Li YJ, Jiang WQ, Huang JJ, Xia ZJ, Huang HQ, Li ZM. The Glasgow Prognostic Score (GPS) as a novel and significant predictor of extranodal natural killer/T-cell lymphoma, nasal type. Am J Hematol. 2013;88:394-9.

11. Kwong YL, Pang AW, Leung AY, Chim CS, Tse E. Quantification of circulating Epstein-Barr virus DNA in NK/T-cell lymphoma treated with the SMILE protocol: diagnostic and prognostic significance. Leukemia. 2014;28:865-70.

12. Yang Y, Zhang YJ, Zhu Y, Cao JZ, Yuan ZY, Xu LM, et al. Prognostic nomogram for overall survival in previously untreated patients with extranodal NK/T-cell lymphoma, nasal-type: a multicenter study. Leukemia. 2015;29:1571-7.

13. Kim SJ, Yoon DH, Jaccard A, Chng WJ, Lim ST, Hong H, et al. A prognostic index for natural killer cell lymphoma after nonanthracycline-based treatment: a multicentre, retrospective analysis. Lancet Oncol. 2016;17:389-400.

14. Yan Z, Huang HQ, Wang XX, Gao Y, Zhang YJ, Bai B, et al. A TNM staging system for nasal NK/T-cell lymphoma. PLoS ONE. 2015;10:e130984.

\section{Affiliations}

Huangming Hong ${ }^{1,2} \cdot$ Yexiong $\mathrm{Li}^{3} \cdot$ Soon Thye $\mathrm{Lim}^{4} \cdot$ Chaoyong Liang ${ }^{5} \cdot \mathrm{He}$ Huang ${ }^{1} \cdot$ Pingyong $\mathrm{Yi}^{6} \cdot \mathrm{Tao} \mathrm{Wu}^{7}$.

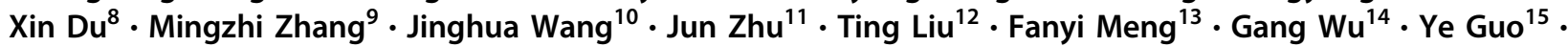
Yuan Zhu ${ }^{16}$. Weili Zhao ${ }^{17}$. Jie Jin ${ }^{18}$. Juan $\mathrm{Li}^{19}$ - Yanming Deng ${ }^{20} \cdot$ Kangsheng $\mathrm{Gu}^{21} \cdot$ Xiangyuan $\mathrm{Wu}^{22}$. Xiaoyan $\mathrm{Ke}^{23} \cdot$ Derong $\mathrm{Xie}^{2} \cdot$ Daren $\mathrm{Lin}^{24} \cdot$ Zhigang Peng ${ }^{25} \cdot$ Junxin $\mathrm{Wu}^{26} \cdot \mathrm{Qing} \mathrm{Liu}^{27} \cdot$ Won Seog Kim ${ }^{28} \cdot$ Tongyu Lin $^{1}$

1 Department of Medical Oncology, Sun Yat-sen University Cancer Center, State Key Laboratory of Oncology in Southern China, and Collaborative Innovation Center of Cancer Medicine, Guangzhou, China
2 Department of Medical Oncology, Sun Yat-sen Memorial Hospital, Sun Yat-Sen University, Guangzhou, China

3 Department of Radiation Oncology, Cancer Institute and Hospital, Chinese Academy of Medical Sciences, Beijing, China 
4 Division of Medical Oncology, National Cancer Centre Singapore, Singapore, Singapore

5 Department of Medical Oncology, Guangxi Medical University Cancer Hospital, Nanning, China

6 Department of Medical Oncology, Hunan Cancer Hospital, Changsha, China

7 Department of Lymphoma-Oncology, Guizhou Cancer Hospital, Guiyang, Guizhou, China

8 Department of Hematology, Guangdong General Hospital, Guangzhou, China

9 Department of Medical Oncology, the First Affiliated Hospital of Zhengzhou University, Zhengzhou, China

10 Department of Medical Oncology, Nanjing General Hospital of Nanjing Military Command, Nanjing, China

11 Department of Lymphoma-Oncology, Peking University Cancer Hospital, Beijing, China

12 Department of Hematology, West China Hospital of Sichuan University, Chengdu, China

13 Department of Hematology, Nanfang Hospital of Southern Medical University, Guangzhou, China

14 Department of Radiation Oncology, Wuhan Union Hospital, Wuhan, China

15 Department of Medical Oncology, Shanghai East Hospital, Tongji University School of Medicine, Shanghai, China

16 Department of Radiation Oncology, Zhejiang Cancer Hospital, Hangzhou, China
17 Department of Hematology, Ruijin Hospital, Shanghai Jiao Tong University School of Medicine, Shanghai, China

18 Department of Hematology, the First Affiliated Hospital Zhejiang University, Hangzhou, China

19 Department of Hematology, the First Affiliated Hospital of Sun Yat-sen University, Guangzhou, China

20 Department of Medical Oncology, the First People's Hospital of Foshan, Foshan, China

21 Department of Medical Oncology, the First Affiliated Hospital of Anhui Medical University, Hefei, China

22 Department of Medical Oncology, the Third Affiliated Hospital of Sun Yat-sen University, Guangzhou, China

23 Department of Hematology, Peking University Third Hospital, Beijing, China

24 Department of Medical Oncology, Jiangmen Central Hospital, Jiangmen, China

25 Department of Medical Oncology, the First Affiliated Hospital of Guangxi Medical University, Nanning, China

26 Department of Radiation Oncology, Fujian Provincial Cancer Hospital, Fuzhou, Fujian, China

27 Department of Epidemiology, Sun Yat-sen University Cancer Center, Guangzhou, China

28 Division of Hematology Oncology, Department of Medicine, Samsung Medical Center, Sungkyunkwan University School of Medicine, Seoul, Korea 